\title{
Proactive interference effects on sentence production
}

\author{
VICTOR S. FERREIRA and CARLA E. FIRATO \\ University of California, San Diego, La Jolla, California
}

\begin{abstract}
Proactive interference refers to recall difficulties caused by prior similar memory-related processing. Information-processing approaches to sentence production predict that retrievability affects sentence form: Speakers may word sentences so that material that is difficult to retrieve is spoken later. In this experiment, speakers produced sentence structures that could include an optional that, thereby delaying the mention of a subsequent noun phrase. This subsequent noun phrase was either (1) conceptually similar to three previous noun phrases in the same sentence, leading to greater proactive interference, or (2) conceptually dissimilar, leading to less proactive interference. Speakers produced more thats (and were more disfluencies) before conceptually similar noun phrases, suggesting that retrieval difficulties during sentence production affect the syntactic structures of sentences that speakers produce.
\end{abstract}

A speaker can communicate an idea with more than one linguistic utterance. ${ }^{1}$ A common (and commonsense) explanation for this observation is that different syntactic structures that convey the same general meaning in fact convey subtly different specific meanings. The implication that naturally follows is that speakers use one syntactic structure rather than another to convey the specific meaning that they intend.

A different explanation for this observation comes from what is here termed the information-processing theory of syntactic formulation (e.g., Bock, 1982, 1987a; Ferreira, 1996; Ferreira \& Dell, 2000). According to this theory, the grammars of languages have evolved to allow different syntactic structures to convey the same general meaning because of the influence of information-processing pressures on sentence production mechanisms. One such pressure arises because the words to be produced in a sentence must be retrieved from lexical memory, an unpredictable and variable process that results in some words being retrieved quickly and others slowly. Importantly, a quickly retrieved word is best mentioned early (so that it need not remain accessible as other sentence material is produced), whereas a slowly retrieved word is best mentioned later (so that the production of other sentence material buys time as it is made accessible). To accommodate this pressure, grammars might allow some flexibility, so

\footnotetext{
We thank Kay Bock, Gary Dell, Fernanda Ferreira, Zenzi Griffin, and John Wixted for valuable discussions and indispensable input on the manuscript. We also thank Caroline Gregorian, Brandy Isaacks, Janet Lee, Ana Molina, Erin Rogers, and Josh Wilson for assistance in materials development and collecting and coding of data. Preparation of this article was supported in part by National Institutes of Health Grant R01 MH64733. Address requests for reprints to V. S. Ferreira, Department of Psychology, University of California, San Diego, La Jolla, CA 92093-0109(e-mail: ferreira@psy.ucsd.edu).
}

that speakers can express the same proposition even when producing a particular word earlier or later.

Indeed, sentence production research supports this general claim, because speakers do tend to produce quickly retrieved words early in sentences and slowly retrieved words later. For example, Bock (1986) showed that semantically primed words were likely to appear earlier in spoken sentences than unprimed words. Other factors that influence sentence production in this manner include (but are not limited to) repetition (Wheeldon \& Monsell, 1992), phonological accessibility (Bock, 1987b), imageability (Bock \& Warren, 1985), and animacy (McDonald, Bock, \& Kelly, 1993).

Ferreira and Dell (2000) extended such effects by showing that the mention of an optional complementizer that in a sentence complement structure (e.g., I knew [that] you had booked a flight for tomorrow) was sensitive to the retrievability of the material produced after the that (e.g., you in the example). Specifically, speakers were more likely to omit the that if the material after it was repeated and was thus more accessible. This suggests that retrievability, at least as it is influenced by repetition, influences whether basic syntactic processes include or omit words like that (see Ferreira, 2001, for evidence that the mention of optional thats is determined by syntactic operations).

Note that alternative approaches that claim that the production of one sentence form rather than another is driven only by meaning differences are inconsistent with this information-processing approach to sentence production (and the evidence that supports it). However, such meaning-based approaches might claim that retrievability is correlated with communicatively relevant information, and that the production of particular sentence forms is driven by representations of such communicatively relevant information. For example, in many languages, speakers tend to produce sentences so that al- 
ready introduced or given information is mentioned before new information (see, e.g., Bock \& Irwin, 1980; MacWhinney \& Bates, 1978; Sridhar, 1988). Perhaps factors such as repetition and semantic priming influence sentence production because, in everyday situations, repetition or semantic (or thematic) relatedness is taken as a cue to givenness, and a representation of givenness then determines the choice of syntactic structure.

This meaning-based explanation of retrievability effects predicts that sentence production should not be sensitive to a retrievability manipulation that is not correlated with any communicative factor. We tested this by assessing the influence of a traditional list-memory effect: proactive interference (see, e.g., Keppel \& Underwood, 1962; see also Wickens, 1972, for review). This well-researched effect refers to the deleterious influence of prior memory performance on current recall, which is greater when the current material is similar in some respect to the previous material. For example, after recalling the names of nine fruits, people have more difficulty recalling the names of three more fruits than they have recalling the names of three items of furniture (e.g., Craik $\&$ Birtwistle, 1971). This suggests that proactive interference arises when currently recalled material is conceptually similar to previously recalled material. Proactive interference can appear as decreased recall accuracy or increased recall latency (e.g., Wixted \& Rohrer, 1993). The retrieval difficulty stems from the decline in the effectiveness of a context-determined recall cue (e.g., fruit) as the size of the set of the applicable items for that recall cue increases (e.g., Watkins \& Watkins, 1975).

In the present experiment, we determined whether proactive interference affects syntactic formulation by looking at the production of the optional that in sentence complement structures. Examples of the materials are shown in Table 1. Sentences began with a main subject that included three noun phrases, then a verb, and then an embedded subject that included one noun phrase. In these sentences, a that may be mentioned immediately before this embedded subject. We manipulated the conceptual similarity of the embedded-subject noun phrase to the three main-subject noun phrases. If proactive interference operates within a sentence, the embedded-subject noun phrase should be retrieved with more difficulty when it is conceptually similar to the main-subject noun phrases. The information-processing theory of syntactic formulation predicts that this slower retrieval of the embedded subject should cause it to be preceded by thats more often. On the other hand, meaning-based accounts do not posit that speakers use optional thats to communicate greater conceptual similarity between a current noun phrase and previously produced noun phrases (e.g., Bolinger, 1972; Elsness, 1984; Thompson \& Mulac, 1991; Yaguchi, 2001). Thus, if speakers produce more thats when the embeddedsubject noun phrase is conceptually similar to the mainsubject noun phrases, this would selectively support the information-processing theory of syntactic formulation.

To elicit sentences, speakers participated in a recallbased sentence production task. When recalling sentences, speakers are known to have good memory for meaning but weak memory for surface form (see, e.g., Bates, Masling, \& Kintsch, 1978; Sachs, 1967). This implies that whereas recall tasks control for the meanings that drive production, the surface forms of produced sentences will vary in a way that allows normal language production effects to emerge (e.g., Bock \& Brewer, 1974; Bock \& Irwin, 1980; Ferreira \& Dell, 2000; Fox Tree \& Meijer, 1999; Lombardi \& Potter, 1992; Potter \& Lombardi, 1990, 1998; Stallings, MacDonald, \& O'Seaghdha, 1998). Also, in addition to measuring speakers' productions for occurrences of that, we measured their fluency and their recall accuracy, to determine other ways in which proactive interference might affect task performance specifically and sentence production generally.

\section{METHOD}

\section{Speakers}

Sixty-four native-English speakers from the UCSD community participated for class credit or cash payment.

\section{Apparatus}

The experiment was administered using PsyScope 1.2.5 (Cohen, MacWhinney, Flatt, \& Provost, 1993), run on Macintosh 6500/250 computers with 17 -in. color monitors. Voice responses were collected with Shure SM10A unidirectional headworn microphones, providing input to Marantz PMD201 cassette recorders (for recording voice responses).

\section{Materials}

Thirty-two sets of four conceptually similar, human-referent noun phrases were designed. To create conceptually similar embeddedsubject sentences, each set was formed into a sentence complement structure with a unique verb, with three noun phrases in the main subject and the remaining noun phrase in the embedded subject (the noun phrase that seemed most typical was the embedded subject). To create conceptually dissimilar sentences, the embedded-subject

Table 1

Two Sample Sentences in Each Condition

Embedded Subject Similarity Condition Conceptually similar

Conceptually dissimilar

Examples

The author, the poet, and the biographer recognized (that) the writer was boring.

The tennis player, the swimmer, and the bowler assumed (that) the golfer was going to make much more money than them.

The author, the poet, and the biographer recognized (that) the golfer was boring.

The tennis player, the swimmer, and the bowler assumed (that) the writer was going to do a story about their careers. 
noun phrases were reassigned among sentences so that each appeared with conceptually dissimilar main subjects. The remainder of each sentence varied to make it sound as reasonable as possible. All sentences were designed with and without the that. Two example sentences of each type are shown in Table 1. The materials are available from the authors upon request.

In addition, 32 filler sentences were created that included four conceptually similar noun phrases from more varied categories and in more varied sentence structures. All sentences were divided into roughly phrase-sized units for presentation.

\section{Design}

We manipulated embedded-subject similarity (conceptually similar or dissimilar to the main-subject noun phrases) and whether the encoded sentence included a that within speakers and items in counterbalanced fashion. Each speaker saw each sentence once, so that each speaker produced eight sentences in each experimental condition. Items were rotated through the four conditions, so that each was presented 16 times per condition across the 64 speakers.

\section{Procedure}

Speakers were tested individually. Each trial began with the message "<get ready $>$." When the experimenter pressed the space bar, the sentence was visually presented one phrase at a time centered on the screen in bold Courier 14-point font, with standard sentence capitalization and standard punctuation. To ensure accurate encoding, the speaker read each phrase out loud, after which the experimenter pressed the space bar to advance to the next phrase. The final phrase of the sentence was followed by a 1-sec delay and then the message "<recall>." The speakers were instructed to recall as much of the just encoded sentence as they could. The sentences were presented in a fixed, randomly determined order, constrained so that no more than three filler or critical trials were presented consecutively. The main experiment was preceded by a practice session that included two sample sentences and a practice block of five sentences. The practice sentences were similar to the fillers.

\section{Scoring and Analysis}

From the tape recordings, an experimentally naive research assistant (1) coded whether each sentence was read aloud accurately upon initial presentation, and (2) transcribed each recalled sentence, including any disfluencies (defined as filled or unfilled pauses, repetitions, corrections, or lengthening). The first author then coded each transcribed sentence for (1) whether it was produced as a sentence complement structure (if the main verb was followed by an embedded subject, before which a that could be mentioned or omitted), (2) number of accurately recalled main-subject noun phrases (in any order), (3) embedded-subject accuracy, (4) the presence or absence of the that, and (5) the presence of at least one disfluency before the end of the embedded-subject noun phrase (which are especially likely to reflect an influence of proactive interference). A sentence was included in the analyses reported below if it was accurately encoded, it was produced as a sentence complement structure, its main subject included at least two of its three noun phrases, and it was produced with the correct embedded-subject noun phrase. Four speakers were excluded from the speaker analyses and two items from the items analyses because these exclusions left one cell of that speaker's or item's design empty. The percentages of scored utterances are shown in Table 2. Other criteria yielded similar results.

The proportions of thats produced and of disfluencies utterances in each experimental condition for each speaker (across items) and each item (across speakers) were calculated, arcsine transformed (Winer, Brown, \& Michels, 1991), and entered into analyses of variance (ANOVAs) with speakers $\left(F_{1}\right)$ and items $\left(F_{2}\right)$ as random factors. All significant effects reached the .05 level or better.

\section{RESULTS AND DISCUSSION}

Along with the percentages of scorable utterances in each condition, Table 2 reports the percentages of scorable utterances that included a that and that included a disfluency before the end of the embedded subject. In addition, the percentages of otherwise scorable utterances that included an erroneous embedded subject are reported, as are the recall accuracies of the that in each embeddedsubject similarity condition.

\section{That Production}

Proactive interference affected that-mention, since speakers produced $5.9 \%$ more thats when the embeddedsubject noun phrase was similar to the main-subject noun phrases in comparison with when it was not, both when the presented sentences contained a that (a $4.9 \%$ difference) and when they did not (a 6.9\% difference). Speakers also produced more thats when the presented sentence contained one. Statistical analyses confirm these observations. The main effect of embedded-subject similarity was significant $\left[F_{1}(1,59)=5.3, M S_{\mathrm{e}}=0.399\right.$; $\left.F_{2}(1,29)=6.4, M S_{\mathrm{e}}=0.272\right]$, as was the effect of whether the presented sentence contained a that $\left[F_{1}(1,59)=22.3\right.$, $\left.M S_{\mathrm{e}}=0.651 ; F_{2}(1,29)=31.6, M S_{\mathrm{e}}=0.335\right]$. The interaction was not significant $\left[F_{1}(1,59)<1, M S_{\mathrm{e}}=0.544\right.$; $\left.F_{2}(1,29)=1.9, M S_{\mathrm{e}}=0.264\right]$, suggesting that proactive interference effects do not differ as a function of whether the presented sentence contains a that. Thus, the pattern of that-mention supports the prediction of the informationprocessing theory of syntactic formulation.

Table 2

Percentages of Scorable Utterances, That Production, Disfluency Rates, Embedded Subject Errors, and That Accuracy for Sentences With Similar or Dissimilar Embedded-Subject Noun Phrases When Sentences Were Encoded With or Without a That

\begin{tabular}{|c|c|c|c|c|c|c|c|c|c|c|c|c|}
\hline \multirow[b]{2}{*}{ Embedded Subject Similarity } & \multicolumn{2}{|c|}{ Scored $\%$} & \multicolumn{3}{|c|}{ That $\%$} & \multicolumn{3}{|c|}{ Disfluency \% } & \multicolumn{3}{|c|}{ Emb. Sub. Err. \% } & \multirow[b]{2}{*}{ That Acc. \% } \\
\hline & That & Zero & That & Zero & $M$ & That & Zero & $M$ & That & Zero & $M$ & \\
\hline Conceptually similar & 54.0 & 50.4 & 88.4 & 73.3 & 80.9 & 61.1 & 58.5 & 59.9 & 23.1 & 23.9 & 23.5 & 58.9 \\
\hline Conceptually dissimilar & 55.0 & 55.2 & 83.5 & 66.4 & 75.0 & 47.7 & 49.3 & 48.5 & 18.9 & 21.3 & 20.1 & 57.9 \\
\hline
\end{tabular}

Notes-Scored \% reports the percentages of utterances evaluated for that mention; that \% reports utterances produced with that; disfluency \% reports utterances with disfluency before the end of the embedded subject; emb. sub. err. \% reports otherwise scorable utterances with an erroneously recalled embedded subject; that acc. \% reports utterances where the that was produced or omitted as originally encoded; that and zero columns refer to conditions where speakers were presented with sentences with or without a that before the embedded subject (respectively). All percentages (except scored \%) are of scorable utterances. 


\section{Disfluencies}

The effect of proactive interference appeared not only as increased that-mention, but also as more disfluencies production. When the embedded-subject noun phrase was similar to the main-subject noun phrases, $11.4 \%$ more utterances included a disfluency before the end of the embedded-subject noun phrase in comparison with when the embedded-subject noun phrase was dissimilar. This was true both when the presented sentences contained a that (a $13.4 \%$ difference) and when they did not (a 9.2\% difference). Speakers were no more disfluencies when the presented sentences contained a that than when they did not (a $0.5 \%$ difference).

The main effect of embedded-subject similarity on the proportion of disfluencies sentences was significant $\left[F_{1}(1,59)=13.3, M S_{\mathrm{e}}=0.557 ; F_{2}(1,29)=7.0, M S_{\mathrm{e}}=\right.$ $0.311]$, whereas the main effect of whether or not the presented sentence contained a that was not $\left[F_{1}(1,59)<1\right.$, $\left.M S_{\mathrm{e}}=0.607 ; F_{2}(1,29)<1, M S_{\mathrm{e}}=0.334\right]$. These factors did not interact $\left[F_{1}(1,59)<1, M S_{\mathrm{e}}=0.480 ; F_{2}(1,29)<1\right.$, $\left.M S_{\mathrm{e}}=0.389\right]$. Thus, an embedded-subject noun phrase that suffers from proactive interference not only causes the increased mention of an optional that, but also causes more disfluencies at sentence positions up to the end of that embedded-subject noun phrase. (The same pattern of effects arises with equal reliability for disfluencies observed only within the embedded-subject noun phrase.)

\section{Embedded-Subject Noun-Phrase Recall}

Standard accounts of proactive interference claim that it affects the success of recall. This predicts that conceptually similar embedded-subjectnoun phrases, which should suffer from proactive interference, may be recalled less accurately than conceptually dissimilar embedded-subject noun phrases. This would provide confirming evidence that conceptually similar embedded-subject noun phrases suffer from greater proactive interference than do conceptually dissimilar ones. However, though there is a tendency in this direction, speakers only recalled embedded-subject noun phrases $3.4 \%$ less accurately when they were conceptually similar in comparison with when they were conceptually dissimilar. This difference was $4.2 \%$ when the presented sentence contained a that and $2.6 \%$ when it did not. Embedded-subject recall accuracy differed by only $1.6 \%$ between the two that-presentation conditions. The main effect of embedded-subject similarity on embedded subject accuracy was marginally significant only by speakers $\left[F_{1}(1,59)=3.3, M S_{\mathrm{e}}=0.302, p<.08 ; F_{2}(1,29)<\right.$ $\left.1, M S_{\mathrm{e}}=0.064\right]$. The main effect of whether a that was presented was not significant $\left[F_{1}(1,59)<1, M S_{\mathrm{e}}=0.414\right.$; $\left.F_{2}(1,29)<1, M S_{\mathrm{e}}=0.086\right]$, nor was the interaction between the two factors $\left[F_{1}(1,59)<1, M S_{\mathrm{e}}=0.321 ; F_{2}(1,29)<1\right.$, $\left.M S_{\mathrm{e}}=0.117\right]$. Thus, although there was a numerical trend for proactive interference to cause less accurate recall of the embedded subject, this effect was not robust.

\section{Memory for That}

A concern is that proactive interference may cause worse memory for the surface features of sentences, which may lead to a differential tendency to mention the that in the two embedded-subject similarity conditions. However, as is shown in Table 2, speakers' memory for the that (the percentage of sentences where speakers produced a that when it was originally encoded or omitted it when it was not) differed only by $1 \%$ (in a direction inconsistent with worse that-memory with greater proactive interference). Two-tailed matched-pairs $t$ tests conducted across speakers and items showed that this difference was not significant $\left[t_{1}(59)=0.29, S E=0.083 ; t_{2}(29)=\right.$ $0.57, S E=0.091]$. Thus, it is unlikely that differential memory for the that in the originally encoded sentence was responsible for the observed effect.

Indeed, speakers' memory for the originally presented that emphasizes the point that the present effects reflect sentence production performance. Although it may seem unsurprising that sentences produced in the context of a memory task are influenced by a memory manipulation, it is important to note how the produced sentences are affected. Specifically, speakers' memory for the that was not affected by the memory manipulation, but rather, speakers simply tended to produce more thats (accurately or inaccurately) when retrieval was more difficult. The information-processing theory of syntactic formulation provides a natural explanation for why that-mention should increase in this way independently of episodic memory for the that: Speakers choose sentence structures that allow the delay of difficult-to-retrieve sentence material.

Finally, to verify that the noun phrases tested in these sentences engendered proactive interference, we conducted an independent list-recall experiment, using a Brown-Peterson-like procedure (see Wickens, 1972). We extracted the four noun phrases from the conceptually similar and dissimilar sentences, along with the noun phrases from the filler sentences. On each trial, 96 new speakers encoded the noun phrases when they were presented one at a time in the same order as in the original sentences. After encoding the four noun phrases, the speakers were presented with a two-digit number between 52 and 98 that was not divisible by three, from which they counted backward by threes at a pace of one number every $800 \mathrm{msec}$ (as determined by short computer-generated beeps). The duration of this distraction task was based on the reading times of the critical sentences within each condition from the main experiment. After the distraction task, the speakers were asked to recall one of the four noun phrases when prompted by the serial position of that noun phrase ("1st," "2nd," "3rd," or "4th"). We manipulated (in counterbalanced fashion) the similarity of the fourth noun phrase to the first three noun phrases as in Experiment 1, as well as the serial position of the noun phrase that the speakers were to recall. We measured recall latency and accuracy; latency analyses were performed only on correct recalls, excluding voice key misdetections ( $8.4 \%$ of correct trials) and latencies outside the range of 500-4,000 msec (3.9\% of correct trials; these exclusions left 12 of the 96 speakers with an empty cell of the design, who were left out of the statistical analyses 
by speakers). If these materials engendered proactive interference, then even in this simple list-recall experiment, the fourth noun phrase (which corresponded to the embedded-subject noun phrases in the main experiment) should be recalled with more difficulty when it was conceptually similar to the first three noun phrases, in comparison with when it was dissimilar.

The results of this independent experiment are shown in Table 3. Proactive interference affected recall latencies, as speakers recalled the fourth noun phrase $218 \mathrm{msec}$ slower when it was conceptually similar to the first three noun phrases than when it was dissimilar. On the other hand, the speakers did not recall the first three noun phrases more slowly (3-msec difference) when the fourth noun phrase was conceptually similar to those noun phrases than when it was dissimilar. Statistical analyses with $2 \times 2$ repeated measures ANOVAs confirmed that the interaction between the serial position of the noun phrase and the similarity of the fourth noun phrase was significant $\left[F_{1}(1,83)=5.0, M S_{\mathrm{e}}=195,986 ; F_{2}(1,31)=4.5\right.$, $\left.M S_{\mathrm{e}}=63,437\right]$. Though recall accuracies differed somewhat among the experimental conditions (see Table 3), no differences were significant (all $p \mathrm{~s}>.1$ ). These results thus confirm that the noun phrases used in the sentences in the main experiment engendered proactive interference.

Overall, the results show that when an embedded-subject noun phrase was conceptually similar to a series of mainsubject noun phrases, it was preceded by a that more often than when it was conceptually dissimilar to those main-subject noun phrases. These results thus support the information-processing theory of syntactic formulation and its claim that language's grammars allow different ways to express the same basic proposition as a result of information-processing pressures that arise during sentence production.

Note that the information-processing approach is not inconsistent with the claim that different sentence forms also differ in meaning. Meaning clearly affects syntactic production (speakers produce either Andy tripped the goalie or Andy was tripped by the goalie, depending on what they mean). The key point here is that the use of a particular utterance form is demonstrably sensitive directly to information-processing pressures, and that such sensitivity cannot be explained solely by meaning-based differences.

It is also worth nothing that that-mention and disfluencies production appear to co-occur, because both were observed under conditions of proactive interference. Indeed, mean proportion of that-mention and mean disfluency rate were reliably correlated within the similar embedded-subject condition both when analyzed by speakers $\left[r=0.3, F_{1}(1,62)=5.9, M S_{\mathrm{e}}=0.063\right]$ and by items $[r=$ $\left.0.5, F_{2}(1,30)=9.9, M S_{\mathrm{e}}=0.031\right]$. (That-mention and disfluency rate were also significantly correlated when measured as differences between the similar and dissimilar embedded-subject conditions.) This suggests that variations in utterance form do not necessarily relieve the information-processing pressures that arise in sentence production, but rather that disfluency and syntactic variation together result from such pressures (see also Bock, 1987b).

Importantly, these sentence production effects were unlikely to have resulted from some feature of meaning that is correlated with proactive interference. Most analyses of the meaning difference between sentence complement structures with and sentence complement structures without a that claim that thats communicate greater conceptual distance between the content of the main and the embedded clauses (e.g., Bolinger, 1972; Elsness, 1984; Thompson \& Mulac, 1991; Yaguchi, 2001). It is unlikely that an embedded-subject noun phrase that is more conceptually similar to a series of main-subject noun phrases would be taken as more conceptually distanced from those main-subject noun phrases.

It may be argued that speakers mentioned thats more often with conceptually similar embedded subjects to assist with any proactive interference that the listener might experience. However, conceptually related words typically facilitate word recognition (see Neely, 1991, for a review). In addition, the speakers did not produce these sentences for listeners. Finally, Ferreira and Dell (2000) showed that speakers do not use thats to avoid difficult-to-comprehend "garden-path" sentences, suggesting that the circumstances under which speakers use thats to assist comprehension processes are limited.

Of course, specific details of performance from the experiment may not generalize straightforwardly to more natural language production situations. In particular, the sizes of the observed differences, the fact that episodic recall was involved, and the fact that the sentences were produced in isolation and were quite unusual all suggest that the specific effects in the experiment are unlikely to have reflected a primary determinant of the sentence choices that speakers make in natural production situations. However, proactive interference is only one kind of memory-

Table 3

Two Sample Word Lists in Each Condition and the Results of the List-Recall Experiment

\begin{tabular}{|c|c|c|c|c|}
\hline \multirow[b]{2}{*}{ Similarity of Fourth Word and Examples } & \multicolumn{2}{|c|}{ Recall of First Three Words } & \multicolumn{2}{|c|}{ Recall of Fourth Word } \\
\hline & Latency (msec) & Accuracy $(\%)$ & Latency (msec) & Accuracy $(\%)$ \\
\hline $\begin{array}{l}\text { Conceptually similar } \\
\text { author, poet, biographer, writer } \\
\text { tennis player, swimmer, bowler, golfer }\end{array}$ & 1,907 & 63.1 & 1,941 & 60.4 \\
\hline $\begin{array}{l}\text { Conceptually dissimilar } \\
\text { author, poet, biographer, golfer } \\
\text { tennis player, swimmer, bowler, writer }\end{array}$ & 1,904 & 57.8 & 1,723 & 59.9 \\
\hline
\end{tabular}


specific factor that could affect sentence production performance. The importance of these results is that they reveal that production mechanisms generally are sensitive to retrievability factors (as claimed by the informationprocessing theory described above), of which proactive interference is only one example. Furthermore, theories of what underlies proactive interference differ in detail, but most agree that it is based on a set-size effect, in that it takes longer to search for (and one is less likely to find) a specific target item in a larger set than in a smaller set. Importantly, such set-size effects arise in semantic generation tasks as well (e.g., Rohrer, Wixted, Salmon, \& Butters, 1995) and share features of codability (Lachman, Shaffer, \& Hennrikus, 1974) and semantic interference effects in word production (e.g., Lupker, 1979), and are thus likely to be relevant to sentence production generally.

Although retrievability has been previously observed to affect the form of the sentences that speakers produce, this is the first demonstration in which retrievability has been manipulated in a way that is obviously unconfounded with meaning features such as givenness. Yet memory directly influenced how sentences were worded. This supports the claim that variations in sentence form come from information-processing pressures that arise during sentence production and not solely from variations in intended meaning.

\section{REFERENCES}

Bates, E., Masling, M., \& Kintsch, W. (1978). Recognition memory for aspects of dialogue. Journal of Experimental Psychology: Human Learning \& Memory, 4, 187-197.

Bock, J. K. (1982). Toward a cognitive psychology of syntax: Information processing contributions to sentence formulation. Psychological Review, 89, 1-47.

Bock, J. K. (1986). Meaning, sound, and syntax: Lexical priming in sentence production. Journal of Experimental Psychology: Learning, Memory, \& Cognition, 12, 575-586.

Bock, J. K. (1987a). Coordinating words and syntax in speech plans. In A. Ellis (Ed.), Progress in the psychology of language (pp. 337-390). London: Erlbaum.

Bock, J. K. (1987b). An effect of the accessibility of word forms on sentence structures. Journal of Memory \& Language, 26, 119-137.

Bock, J. K., \& BREWER, W. F. (1974). Reconstructive recall in sentences with alternative surface structures. Journal of Experimental Psychology, 103, 837-843.

Bock, J. K., \& IRWIN, D. E. (1980). Syntactic effects of information availability in sentence production. Journal of Verbal Learning \& Verbal Behavior, 19, 467-484.

Bock, J. K., \& WARren, R. K. (1985). Conceptual accessibility and syntactic structure in sentence formulation. Cognition, 21, 47-67.

Bolinger, D. (1972). That's that. The Hague: Mouton.

Cohen, J., MacWhinney, B., Flatt, M., \& Provost, J. (1993). PsyScope: An interactive graphic system for designing and controlling experiments in the psychology laboratory using Macintosh computers. Behavior Research Methods, Instruments, \& Computers, 25, 257-271.

Craik, F. I. [M.], \& BirTwist Le, J. (1971). Proactive inhibition in free recall. Journal of Experimental Psychology, 91, 120-123.

ELSNESS, J. (1984). That or zero? A look at the choice of object clause connective in a corpus of American English. English Studies, 65, 519-533.

Ferreira, V. S. (1996). Is it better to give than to donate? Syntactic flexibility in language production. Journal of Memory \& Language, 35, 724-755.

Ferreira, V. S. (2001, March). Isolating the syntactic choice. Paper presented at the 14th Annual CUNY Conference on Human Sentence Processing, Philadelphia.
Ferreira, V. S., \& Dell, G. S. (2000). Effect of ambiguity and lexical availability on syntactic and lexical production. Cognitive Psychology, 40, 296-340.

Fox Tree, J. E., \& MeiJer, P. J. A. (1999). Building syntactic structure in speaking. Journal of Psycholinguistic Research, 28, 71-92.

KePPEL, G., \& UNDERWOOd, B. J. (1962). Proactive inhibition in shortterm retention of single items. Journal of Verbal Learning \& Verbal Behavior, 1, 153-161.

Lachman, R., Shaffer, J. P., \& Hennrikus, D. (1974). Language and cognition: Effects of stimulus codability, name-word frequency, and age of acquisition on lexical reaction time. Journal of Verbal Learning \& Verbal Behavior, 13, 613-625.

Lombardi, L., \& Potter, M. C. (1992). The regeneration of syntax in short-term memory. Journal of Memory \& Language, 31, 713-733.

LUPKER, S. J. (1979). The semantic nature of response competition in the picture-word interference task. Memory \& Cognition, 7, 485-495.

MacWhinney, B., \& Bates, E. (1978). Sentential devices for conveying givenness and newness: A cross-cultural developmental study. Journal of Verbal Learning \& Verbal Behavior, 17, 539-558.

McDonald, J. L., Bock, K., \& Kelly, M. H. (1993). Word and world order: Semantic, phonological, and metrical determinants of serial position. Cognitive Psychology, 25, 188-230.

NeELy, J. H. (1991). Semantic priming effects in visual word recognition: A selective review of current findings and theories. In G. H. D. Besner (Ed.), Basic processes in reading: Visual word recognition (pp. 264-336). Hillsdale, NJ: Erlbaum.

Potter, M. C., \& Lombardi, L. (1990). Regeneration in the short-term recall of sentences. Journal of Memory \& Language, 29, 633-654.

PotTer, M. C., \& Lombardi, L. (1998). Syntactic priming in immediate recall of sentences. Journal of Memory \& Language, 38, 265-282.

Rohrer, D., Wixted, J. T., SAlmon, D. P., \& Butters, N. (1995). Retrieval from semantic memory and its implications for Alzheimer's disease. Journal of Experimental Psychology: Learning, Memory, \& Cognition, 21, 1127-1139.

SACHS, J. S. (1967). Recognition memory for syntactic and semantic aspects of connected discourse. Perception \& Psychophysics, 2, 437-442.

Sridhar, S. N. (1988). Cognition and sentence production: A crosslinguistic study. New York: Springer-Verlag.

Stallings, L. M., MacDonald, M. C., \& O'Seaghdha, P. G. (1998). Phrasal ordering constraints in sentence production: Phrase length and verb disposition in heavy-NP shift. Journal of Memory \& Language, 39, 392-417.

Thompson, S. A., \& Mulac, A. (1991). The discourse conditions for the use of the complementizer that in conversational English. Journal of Pragmatics, 15, 237-251.

WATKIns, O. C., \& WATKIns, M. J. (1975). Buildup of proactive inhibition as a cue-overload effect. Journal of Experimental Psychology: Human Learning \& Memory, 1, 442-452.

WheEldon, L. R., \& Monsell, S. (1992). The locus of repetition priming of spoken word production. Quarterly Journal of Experimental Psychology, 44A, 723-761.

WiCKENS, D. D. (1972). Characteristics of word encoding. In A. W. Melton \& E. Martin (Eds.), Coding processes in human memory (pp. 195215). Washington, DC: Winston.

Winer, B. J., Brown, D. R., \& Michels, K. M. (1991). Statisticalprinciples in experimental design (3rd ed.). New York: McGraw-Hill.

Wixted, J. T., \& Rohrer, D. (1993). Proactive interference and the dynamics of free recall. Journal of Experimental Psychology: Learning, Memory, \& Cognition, 19, 1024-1039.

YAGUCHI, M. (2001). The function of the non-deictic that in English. Journal of Pragmatics, 33, 1125-1155.

\section{NOTE}

1. "An idea can be communicated by a speaker with more than one linguistic utterance." "More than one linguistic utterance can be used by a speaker to communicate an idea." And so forth.

(Manuscript received April 17, 2001; revision accepted for publication December 4, 2001.) 